\title{
LHCb spectroscopy results
}

\author{
Roberta Cardinale*, on behalf of the LHCb Collaboration \\ University and INFN of Genova \\ E-mail: roberta.cardinale@cern.ch
}

Spectroscopy studies are an essential part of the LHCb experiment physics programme. The unique collected data samples and the excellent performance of the LHCb detector enable precision studies in the spectroscopy sector. These measurements provide tests for effective theories of the strong interaction. The latest results obtained by the LHCb experiment in the standard and exotic spectroscopy sectors are presented.

Sixth Annual Conference on Large Hadron Collider Physics (LHCP2018)

4-9 June 2018

Bologna, Italy

${ }^{*}$ Speaker. 


\section{Observation of the doubly-charmed $\Xi_{c c}^{++}$baryon}

Doubly heavy baryons, predicted by the quark model, represent a mostly unexplored region where theoretical predictions have no support from the experimental point of view. In particular for the $\Xi_{c c}^{++}$baryon, many predictions for its mass are available in a well constrained range between $3.5-3.7 \mathrm{GeV} / c^{2}$ separated by only few $\mathrm{MeV} / c^{2}$ to the $\Xi_{c c}^{+}$state, due to approximate isospin symmetry. Different theoretical predictions are available also for its lifetime showing a large ambiguity between $150-1550 \mathrm{fs}$.

Experimentally, the SELEX experiment claimed the observation of a peak in the $K^{-} \pi^{+} \Lambda_{c}^{+}$ and in the $p D^{+} K^{-}$mass spectra $[1,2]$. This excess has been identified as the singly charged $\Xi_{c c}^{+}$ state. SELEX measured its mass to be $M=3518.7 \pm 1.7 \mathrm{MeV} / c^{2}$ and an unexpected short lifetime $\tau<33 \mathrm{fs}$ at $90 \% \mathrm{CL}$ which is not compatible with theoretical predictions. The $\Xi_{c c}^{+}$state has not been confirmed by other experiments, such as BaBar [3], Belle [4] and LHCb [5].

Recently, the $\mathrm{LHCb}$ experiment performed an analysis using data samples both from 2012 at a centre-of-mass energy of $8 \mathrm{TeV}$ and from 2016 at $13 \mathrm{TeV}$, searching for the isospin partner of the SELEX claimed state, the $\Xi_{c c}^{++}$baryon, in $\Lambda_{c}^{+} K^{-} \pi^{+} \pi^{-}$where the $\Lambda_{c}^{+}$is reconstructed in the $p K^{-} \pi^{+}$final state [6]. The invariant mass plots for the two data samples are shown in Figure 1 where highly significant peaks ( $7.6 \sigma$ for 2012 and $12.9 \sigma$ for 2016 data samples) are visible in both samples.
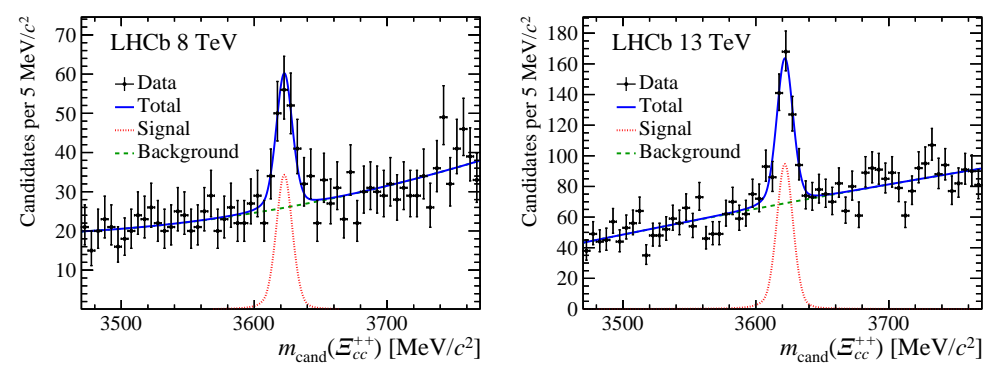

Figure 1: Invariant mass distribution of $\Lambda_{c}^{+} K^{-} \pi^{+} \pi^{-}$candidates with fit projections overlaid for 2012 (left) and 2016 (right) data samples.

The 2016 data sample is used to measure the mass of the $\Xi_{c c}^{++}$state giving a value of $m\left(\Xi_{c c}^{++}\right)=$ $3621.40 \pm 0.72$ (stat) \pm 0.31 (syst) $\mathrm{MeV} / c^{2}$. This value is consistent with the theoretical range of predictions while it is shifted of the order of $100 \mathrm{MeV} / c^{2}$ from the SELEX measurement which is too large for an isospin splitting.

Measurements of the properties of the $\Xi_{c c}^{++}$state are crucial, in particular its lifetime, to establish the weak nature of its decay and to compare the short lifetime measured by SELEX and the theoretical predictions. A lifetime measurement is performed at the LHCb experiment [7] using a data sample corresponding to an integrated luminosity of $1.7 \mathrm{fb}^{-1}$ at $\sqrt{s}=13 \mathrm{TeV}$ reconstructing the $\Xi_{c c}^{++}$state in $\Lambda_{c}^{+} K^{-} \pi^{+} \pi^{+}$relative to the control channel $\Lambda_{b}^{0} \rightarrow \Lambda_{c}^{+} \pi^{-} \pi^{+} \pi^{-}$. The invariant mass spectra for the signal and the control channels are shown in Figure 2. The unbinned maximum likelihood fit of the background-subtracted $\Xi_{c c}^{++}$decay distribution, shown in Figure 2, gives $\tau_{\Xi_{c c}^{++}}=0.256_{-0.022}^{+0.224}($ stat $) \pm 0.014$ (syst $)$ ps, compatible with a weak decay. Other decay modes of the $\Xi_{c c}^{++}$state are currently under study and the search for other doubly-charmed baryon states 

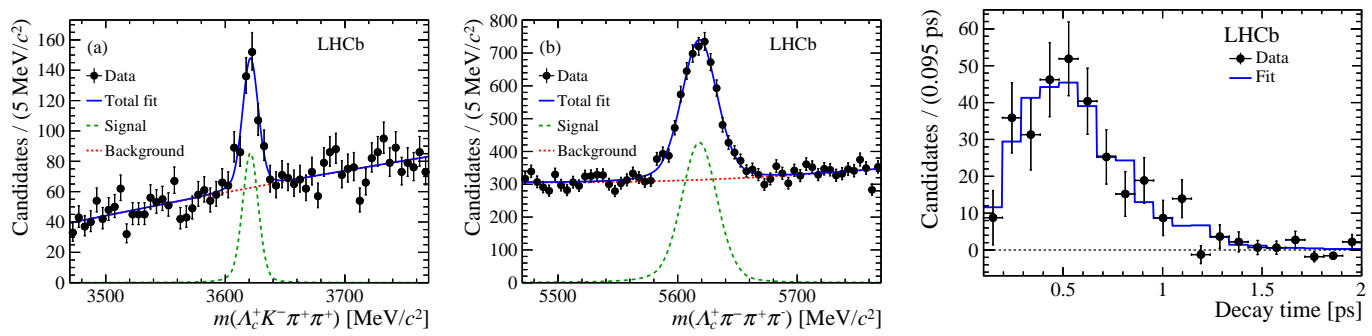

Figure 2: Fit to the invariant-mass distributions of (a) $\Xi_{c c}^{++} \rightarrow \Lambda_{c}^{+} K^{-} \pi^{+} \pi^{+}$and (b) $\Lambda_{b}^{0} \rightarrow \Lambda_{c}^{+} \pi^{-} \pi^{+} \pi^{-}$ candidates. Background-subtracted decay-time distribution of $\Xi_{c c}^{++}$candidates with the rate-averaged fit result across each decay-time bin shown as the continuous line (right).

opened. Recently a confirmation of the $\Xi_{c c}^{++}$baryon through the $\Xi_{c c}^{++} \rightarrow \Xi_{c}^{+} \pi^{+}$decay has been obtained by $\mathrm{LHCb}[8]$.

\section{Observation of a new $\Xi_{b}^{-}$resonance}

Numbers of excited $b$-baryons have already been discovered, such as the $\Xi_{b}^{\prime}(5935)^{-}$and the $\Xi_{b}^{*}(5955)^{-}$states which are the first radial and orbital excitations of the ground $\Xi_{b}^{-}$state by the LHCb experiment [9], or the first orbital excitation of the $\Xi_{b}^{0}$ baryon, the $\Xi_{b}^{*}(5945)^{0}$ state, by the CMS experiment [10] and confirmed by the LHCb experiment [11]. The higher excited states are expected to be above the $\Lambda_{b}^{0} K$ threshold allowing this final state to be used for excited $b$-baryon states searches. At LHCb, searches for excited $\Xi_{b}$ states are performed using both $\Lambda_{b}^{0} K^{-}$ and $\Xi_{b}^{0} \pi^{-}$final states using the full Run1 data sample (corresponding to an integrated luminosity of $1.0 \mathrm{fb}^{-1}$ at $7 \mathrm{TeV}$ and $2.0 \mathrm{fb}^{-1}$ at $8 \mathrm{TeV}$ ) plus $1.5 \mathrm{fb}^{-1}$ of Run2 data at $13 \mathrm{TeV}$ [12]. The $\Xi_{b}^{0}$ is reconstructed via the semileptonic $\Lambda_{c}^{+} \mu^{-} X$ decay, while the $\Lambda_{b}^{0}$ is reconstucted both via the hadronic $\Lambda_{c}^{+} \pi^{-}$decay and via the semileptonic $\Lambda_{c}^{+} \mu^{-} X$ decay. The three invariant mass spectra for the three considered final states are shown for the two considered datasets in Figure 3. A peak is clearly visible in all mass spectra with a significance of $9.2 \sigma, 25 \sigma$ and $7.9 \sigma$ for the semileptonic $\Xi_{b}^{0}$, the semileptonic $\Lambda_{b}^{0}$ and the hadronic $\Lambda_{b}^{0}$ decays, respectively. Using the $\Lambda_{b}^{0}$ hadronic channel, which has the best resolution $\left(\sim 2 \mathrm{MeV} / c^{2}\right)$, the mass and the width of this new state are measured to be $m_{\Xi_{b}(6227)^{-}}=6226.9 \pm 2.0 \pm 0.3 \pm 0.2 \mathrm{MeV} / c^{2}$ and $\Gamma_{\Xi_{b}(6227)^{-}}=18.1 \pm 5.4 \pm 1.8 \mathrm{MeV} / c^{2}$ where the first uncertainty is statistical, the second is systematic, and the third, on $m_{\Xi_{b}(6227)^{-}}$, comes from the uncertainty of the $\Lambda_{b}^{0}$ baryon mass. Production ratios are also measured using the semileptonic modes. The measured proprierties are consistent with expectations of either the $\Xi_{b}(1 P)$ or the $\Xi_{b}(2 S)$ states.

\section{Studies of the $\chi_{c 1}$ and the $\chi_{c 2}$ resonance parameters with the decays $\chi_{c 1, c 2} \rightarrow J / \psi \mu^{+} \mu^{-}$}

Studies of the properties and production of quarkonia at hadron colliders provide an important test of the quantum chromodynamic models. Most of the studies of the $\chi_{c 1, c 2}$ states have been performed using radiative $\chi_{c 1, c 2} \rightarrow J / \psi \gamma$ decays. At LHCb, the first observation of the 

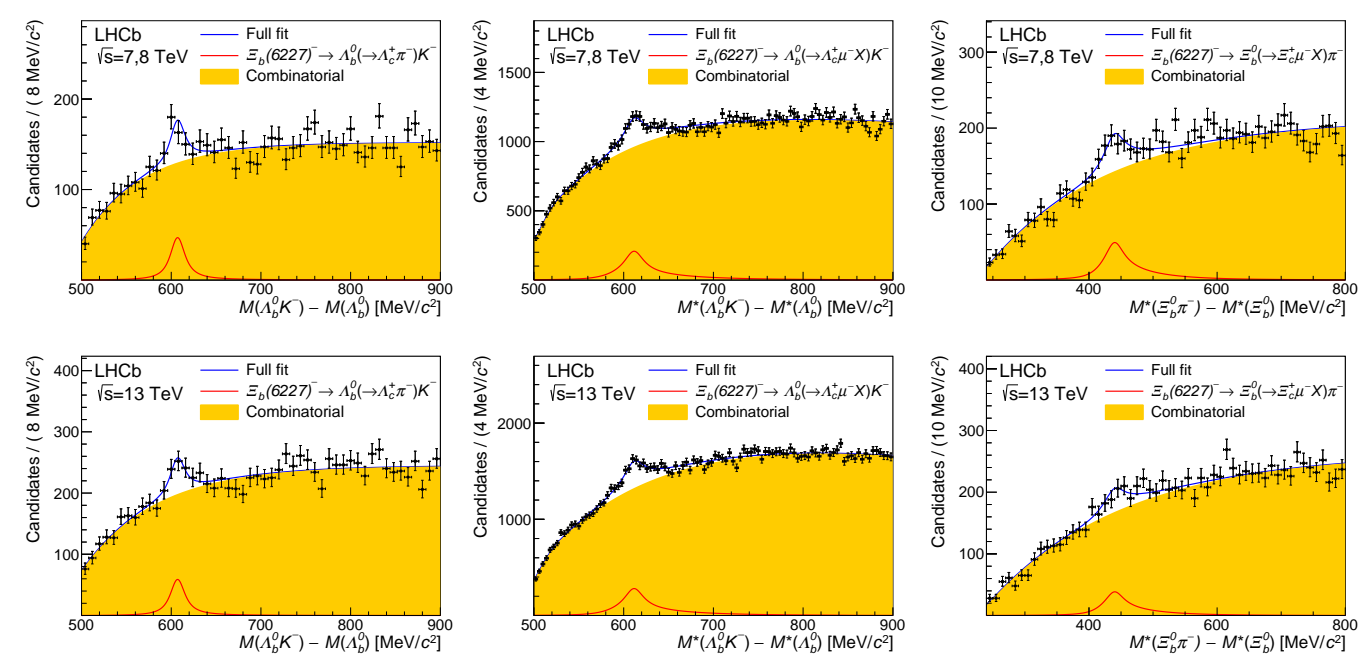

Figure 3: Invariant mass difference spectra for $\Xi_{b}(6227)^{-}$candidates for the three considered final states. Top row is for $7,8 \mathrm{TeV}$ and bottom for $13 \mathrm{TeV}$ data.

$\chi_{c 1, c 2} \rightarrow J / \psi \mu^{+} \mu^{-}$decays has been performed using the full Run1 data sample and data from Run 2 corresponding to an integrated luminosity of $1.9 \mathrm{fb}^{-1}$ at $13 \mathrm{TeV}$ [13]. The observed Dalitz decay has the clear advantage of a clean signature at hadron colliders with respect to the radiative decay having four muons in the final state. The mass distribution for the selected candidates is shown in Figure 4. The masses of these states are measured to be $m\left(\chi_{c 1}\right)=3510.71 \pm 0.04 \pm 0.09 \mathrm{MeV} / c^{2}$

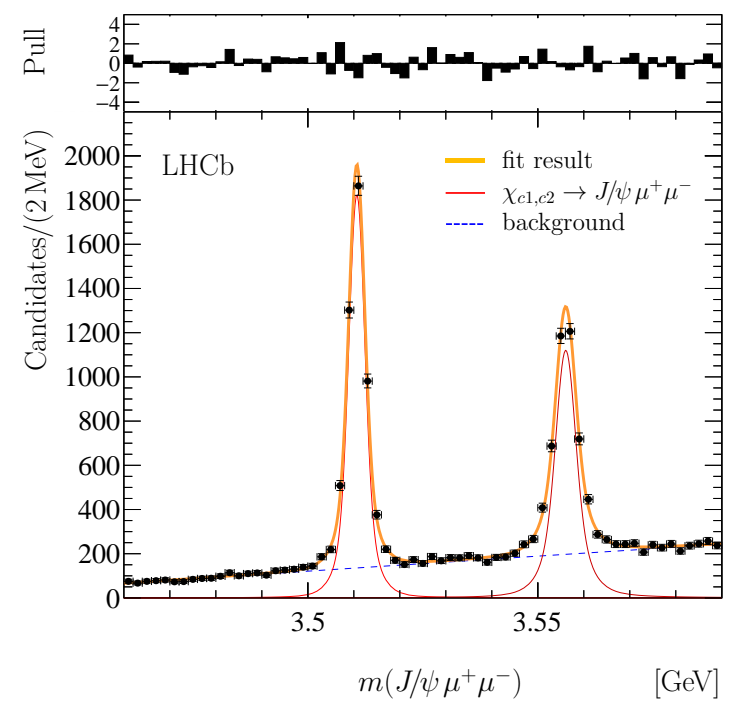

Figure 4: Mass spectrum for selected $J / \psi \mu^{+} \mu^{-}$candidates.

and $m\left(\chi_{c 2}\right)=3556.10 \pm 0.06 \pm 0.11 \mathrm{MeV} / c^{2}$. The momentum-scale uncertainties which are the dominant systematic uncertainty in the mass measurements largely cancel in the mass difference $m\left(\chi_{c 2}\right)-m\left(\chi_{c 1}\right)=45.39 \pm 0.07 \pm 0.03 \mathrm{MeV} / c^{2}$. The natural width of the $\chi_{c 2}$ meson is measured to be $\Gamma\left(\chi_{c 2}\right)=2.10 \pm 0.20$ (stat) \pm 0.02 (syst) $\mathrm{MeV} / c^{2}$. These results are in good agreement with and have comparable precision to the current world averages. This result opens up a new avenue 
for hadron spectroscopy at the LHC with the possibility of the measurement of the production of the $\chi_{c 1}$ and $\chi_{c 2}$ states at low transverse momentum and of other heavy-flavour states when larger data samples will be available.

\section{Search for weakly decaying b-flavoured pentaquarks}

The observation of two states consistent with two charmonium pentaquark states ( $c \bar{c} u u d$ ) by the LHCb experiment in $\Lambda_{b}^{0} \rightarrow J / \psi p K^{-}$and $\Lambda_{b}^{0} \rightarrow J / \psi p \pi^{-}$decays raised the interest of searches of other pentaquark states $[14,15,16]$. The LHCb experiment searched for weakly-decaying pentaquark states containing a $b$-quark [17]. According to the Skyrme model [18], heavy quarks give tightly bound pentaquarks. Using modes which include a $J / \psi$ in the final state which have large efficiencies and reduced background, searches of four different states have been performed below the corresponding strong decay threshold mass: $P_{B^{0} p}^{+} \rightarrow J / \psi K^{+} \pi^{-} p$ in $4668-6220 \mathrm{MeV} / c^{2}$, $P_{\Lambda_{b}^{0} \pi^{-}}^{-} \rightarrow J / \psi K^{-} \pi^{-} p$ in $4668-5760 \mathrm{MeV} / c^{2}, P_{\Lambda_{b}^{0} \pi^{+}}^{+} \rightarrow J / \psi K^{-} \pi^{+} p$ in $4668-5760 \mathrm{MeV} / c^{2}$ and $P_{B_{s}^{0} p}^{+} \rightarrow J / \psi \phi p$ in $5055-6305 \mathrm{MeV} / c^{2}$. The states are labelled with a subscript indicating the final states the pentaquark would predominantly decay into if it had sufficient mass to decay strongly in these states. Measurement of the product of production cross section and branching ratio with respect to the $\Lambda_{b}^{0} \rightarrow J / \psi p K^{-}$decay is performed but no evidence for signal is found and CL limits at $90 \%$ are set (see Figure 5).
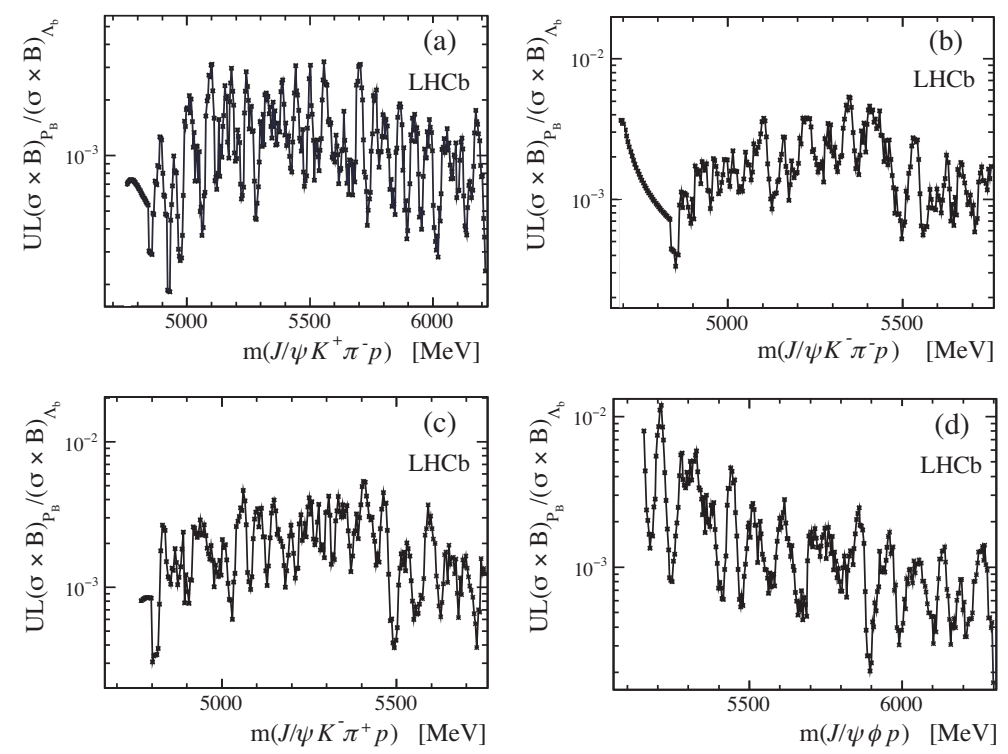

Figure 5: Upper limits at $90 \% \mathrm{CL}$ on the product of production cross section and branching ratio with respect to the $\Lambda_{b}^{0} \rightarrow J / \psi p K^{-}$decay for (a) $J / \psi K^{+} \pi^{-} p$, (b) $J / \psi K^{-} \pi^{-} p$, (c) $J / \psi K^{-} \pi^{+} p$ and (d) $J / \psi \phi p$ final states.

\section{Search for beautiful tetraquarks in the $\Upsilon(1 S) \mu^{+} \mu^{-}$invariant mass spectrum}

A search for a possible exotic meson state composed of two $b$ and two $\bar{b}$ quarks, $X_{b b \bar{b} \bar{b}}$, has been performed at LHCb in the $\Upsilon(1 S) \mu^{+} \mu^{-}$invariant mass spectrum using the whole Run1 dataset 
and $3.3 \mathrm{fb}^{-1}$ of Run2 data sample [19]. Several theoretical models predict the mass and the width of an exotic $X_{b b \bar{b} \bar{b}}$ state. In particular the mass is indicated to be in the range $18.4-18.8 \mathrm{GeV} / c^{2}$, below the $\eta_{b} \eta_{b}$ invariant mass. No significant excess is seen for any mass hypothesis in the range $[17.5,20.0] \mathrm{GeV} / c^{2}$ and upper limits at $95 \% \mathrm{CL}$ on the production cross sections times the branching fractions, $\sigma(p p \rightarrow X) \times \mathscr{B}\left(X \rightarrow \Upsilon(1 S) \mu^{+} \mu^{-}\right) \times \mathscr{B}\left(\Upsilon(1 S) \rightarrow \mu^{+} \mu^{-}\right)$are set (see Figure 6).
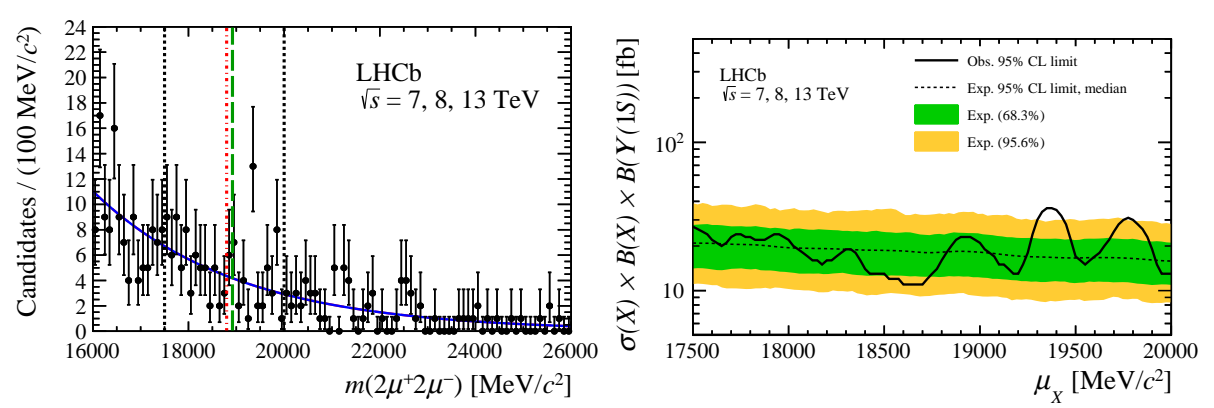

Figure 6: (Left) Distribution of $m\left(2 \mu^{+} 2 \mu^{-}\right)$. The dotted black lines indicate the range in which limits are set on the product of the $X$ production cross section and branching fractions. The dash-dotted red and longdashed green lines show the positions of the $\eta_{b} \eta_{b}$ and $\Upsilon(1 S) \Upsilon(1 S)$ thresholds, respectively. (Right) The 95\% $\mathrm{CL}$ on the production cross sections times the branching fractions as a function of the $X$ mass hypothesis.

\section{Search for dibaryon states}

A hypothetical charmed dibaryon state $D_{c}^{+}=[c d][u d][u d]$ could be produced in $\Lambda_{b}^{0} \rightarrow \bar{p} D_{c}^{+}(\rightarrow$ $\left.\Lambda_{c}^{+} \pi^{-} p\right)$ decays. The $D_{c}^{+}$dibaryon decay could proceed via quark rearrangement to the final state $p \Lambda_{c}^{+} \pi^{-}$through the $\Sigma_{c}^{0}$ intermediate state or via a lighter pentaquark state $P_{c}(\bar{u}[c d][u d])$. The LHCb experiment observed for the first time the $\Lambda_{b}^{0} \rightarrow \Lambda_{c}^{+} p \bar{p} \pi^{-}$decay using the full Run1 data [20]. Its branching fraction relative to the $\Lambda_{b}^{0} \rightarrow \Lambda_{c}^{+} \pi^{-}$decay channel including the ones with $\Sigma_{c}^{0}$ and $\Sigma_{c}^{* 0}$ intermediate resonances are measured. No evidence for any dibaryon signal is observed in the $\Lambda_{c}^{+} p \bar{p} \pi^{-}$final state (see Figure 7).
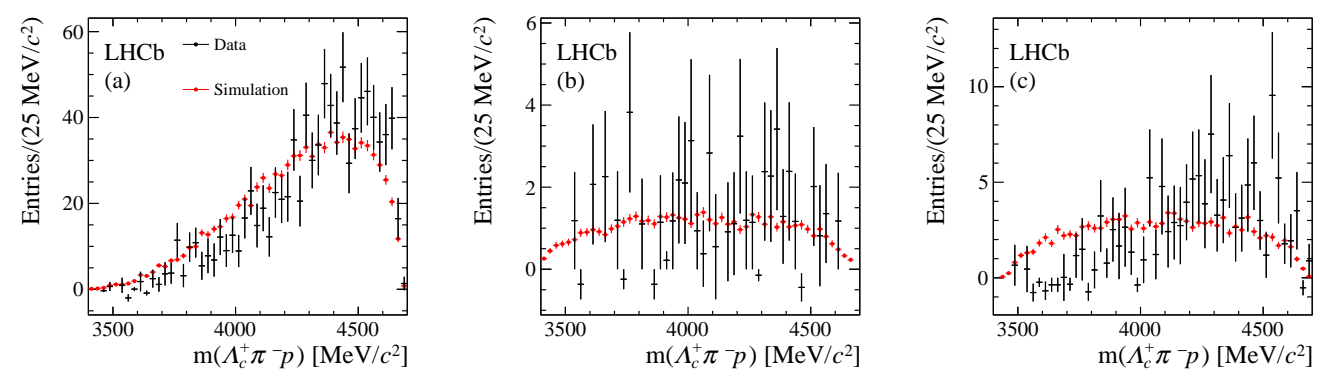

Figure 7: Background-subtracted mass spectrum of the $\Lambda_{c}^{+} \pi^{-} p$ system from the decay $\Lambda_{b}^{0} \rightarrow \Lambda_{c}^{+} p \bar{p} \pi^{-}$in (a) the full $\Lambda_{c}^{+} \pi^{-}$mass spectrum, (b) the $\Sigma_{c}^{0}$ resonance and (c) the $\Sigma_{c}^{* 0}$ resonance. No evident peaking shapes are visible. 


\section{References}

[1] M. Mattson et al. [SELEX Collaboration], Phys. Rev. Lett. 89 (2002) 112001 doi:10.1103/PhysRevLett.89.112001 [hep-ex/0208014].

[2] A. Ocherashvili et al. [SELEX Collaboration], Phys. Lett. B 628 (2005) 18 doi:10.1016/j.physletb.2005.09.043 [hep-ex/0406033].

[3] B. Aubert et al. [BaBar Collaboration], Phys. Rev. D 74 (2006) 011103 doi:10.1103/PhysRevD.74.011103 [hep-ex/0605075].

[4] R. Chistov et al. [Belle Collaboration], Phys. Rev. Lett. 97 (2006) 162001 doi:10.1103/PhysRevLett.97.162001 [hep-ex/0606051].

[5] R. Aaij et al. [LHCb Collaboration], JHEP 1312 (2013) 090 doi:10.1007/JHEP12(2013)090 [arXiv:1310.2538 [hep-ex]].

[6] R. Aaij et al. [LHCb Collaboration], Phys. Rev. Lett. 119 (2017) 112001 doi:10.1103/PhysRevLett.119.112001 [arXiv:1707.01621 [hep-ex]].

[7] R. Aaij et al. [LHCb Collaboration], Phys. Rev. Lett. 121 (2018) 052002 doi:10.1103/PhysRevLett.121.052002 [arXiv:1806.02744 [hep-ex]].

[8] R. Aaij et al. [LHCb Collaboration], arXiv:1807.01919 [hep-ex].

[9] R. Aaij et al. [LHCb Collaboration], Phys. Rev. Lett. 114 (2015) 062004 doi:10.1103/PhysRevLett.114.062004 [arXiv:1411.4849 [hep-ex]].

[10] S. Chatrchyan et al. [CMS Collaboration], Phys. Rev. Lett. 108 (2012) 252002 doi:10.1103/PhysRevLett.108.252002 [arXiv:1204.5955 [hep-ex]].

[11] R. Aaij et al. [LHCb Collaboration], JHEP 1605 (2016) 161 doi:10.1007/JHEP05(2016)161 [arXiv:1604.03896 [hep-ex]].

[12] R. Aaij et al. [LHCb Collaboration], Phys. Rev. Lett. 121 (2018) 072002 doi:10.1103/PhysRevLett.121.072002 [arXiv:1805.09418 [hep-ex]].

[13] R. Aaij et al. [LHCb Collaboration], Phys. Rev. Lett. 119 (2017) 221801 doi:10.1103/PhysRevLett.119.221801 [arXiv:1709.04247 [hep-ex]].

[14] R. Aaij et al. [LHCb Collaboration], Phys. Rev. Lett. 115 (2015) 072001 doi:10.1103/PhysRevLett.115.072001 [arXiv:1507.03414 [hep-ex]].

[15] R. Aaij et al. [LHCb Collaboration], Phys. Rev. Lett. 117 (2016) 082002 doi:10.1103/PhysRevLett.117.082002 [arXiv:1604.05708 [hep-ex]].

[16] R. Aaij et al. [LHCb Collaboration], Phys. Rev. Lett. 117 (2016) 082003 doi:10.1103/PhysRevLett.118.119901; Phys. Rev. Lett. 117 (2016) 109902 10.1103/PhysRevLett.117.082003; Phys. Rev. Lett. 118 (2017) 119901 10.1103/PhysRevLett.117.109902 [arXiv:1606.06999 [hep-ex]].

[17] R. Aaij et al. [LHCb Collaboration], Phys. Rev. D 97 (2018) 032010 doi:10.1103/PhysRevD.97.032010 [arXiv:1712.08086 [hep-ex]].

[18] T. H. R. Skyrme, Proc. Roy. Soc. Lond. A 260 (1961) 127. doi:10.1098/rspa.1961.0018

[19] R. Aaij et al. [LHCb Collaboration], arXiv:1806.09707 [hep-ex].

[20] R. Aaij et al. [LHCb Collaboration], Phys. Lett. B 784 (2018) 101 doi:10.1016/j.physletb.2018.07.033 [arXiv:1804.09617 [hep-ex]]. 